\title{
Composition of Saliency Metrics for Pruning with a Myopic Oracle
}

\author{
Kaveena Persand \\ School of Computer Science \\ Trinity College Dublin \\ Dublin 2, Ireland \\ persandk@tcd.ie
}

\author{
Andrew Anderson \\ School of Computer Science \\ Trinity College Dublin \\ Dublin 2, Ireland \\ aanderso@tcd.ie
}

\author{
David Gregg \\ School of Computer Science \\ Trinity College Dublin \\ Dublin 2, Ireland \\ david.gregg@cs.tcd.ie
}

\begin{abstract}
The cost of Convolutional Neural Network (CNN) inference can be reduced by pruning weights from a trained network, eliminating computations while preserving the predictive accuracy up to some threshold. While many heuristic saliency metrics have been proposed to guide this process, the quality of pruning decisions made by any one metric is highly contextsensitive. Some metrics make excellent pruning decisions for one network, while making poor decisions for other networks.

Traditionally, a single heuristic saliency metric is used for the entire pruning process. We show how to compose a set of these saliency metrics to form a much more robust (albeit still heuristic) saliency. The key idea is to exploit the cases where the different base metrics do well, and avoid the cases where they do poorly by switching to a different metric. With an experimental evaluation of channel pruning on several popular CNNs on the CIFAR-10 and CIFAR-100 datasets, we show that the composite saliency metrics derived by our method consistently outperform all of the individual constituent metrics.
\end{abstract}

Index Terms-Machine Learning, Convolutional Neural Networks, Pruning

\section{Motivation}

The fundamental action of pruning is to identify a subset of the weights of a neural network which can be removed (pruned) without damaging the predictive accuracy of the network by more than a user-supplied threshold. This threshold is application specific: in optical character recognition, for example, the acceptable drop in accuracy may be much larger than in real-time pedestrian detection.

In order to find optimal prunings of a neural network, we can use a simple, but computationally expensive approach: considering all possible subsets of unpruned weights, prune each subset independently and measure the change in accuracy to select the optimal set of weights to remove. In practice, pruning is not done in this way due to the prohibitive cost. Instead a pruning scheme which determines when, how many, and which weights need to be removed is used.

Many pruning schemes use a saliency metric to determine which subsets of weights are least likely to damage the network[1], [2], [3], [4], [5], [6], [7]. The saliency metric is a heuristic used to efficiently rank different subsets of weights. A wide variety of heuristic saliency metrics have been proposed over decades of research in artificial intelligence[2], [5], [6], [7], [8]. Each of these heuristic saliency metrics may perform better or worse in context, with no one metric being clearly superior. When pruning a neural network, the data scientist must often resort to simple rules of thumb or guesswork to select an appropriate heuristic to guide the pruning process.

\section{A. Contributions}

We propose a method to derive a composite saliency metric which can avoid poor choices made by otherwise effective constituent saliency metrics. Our approach uses a myopic oracle to decide which of a fixed set of constituent metrics should be active at every step of the pruning process. As the predictive power of the constituent metrics waxes and wanes, our approach dynamically switches between metrics so that the most appropriate metric is guiding the process at all points.

We make the following principal contributions:

- We demonstrate that different metrics perform differently on different networks.

- We show how to compose different saliency metrics automatically (the myopic oracle).

- We experimentally investigate fusion of state-of-the-art saliency metrics.

\section{BACKGROUND}

The goal of pruning is to remove the largest structured or unstructured group of weights without damaging the performance of the network. Pruning schemes can be categorised roughly as either saliency based or penalty term based. Penalty term based schemes modify the cost function to prune weights. Saliency metric based schemes use a metric to identify which weights or set of weights are least important to the network. While early work on saliency metrics focused on using fully trained networks [7], [9], [5], [6], recent work has shown that saliency metrics can successfully be used to remove parameters from the network at different stages of the training process [10], [11].

In this paper, we focus on saliency metrics for coarse granularity pruning, i.e., removing entire convolution channels from a network.

\section{A. Classification of Metrics}

In the following treatment, we refer to the $c$ th subset of weights as $W_{c}$, with corresponding output activations $A_{c}$. 
Weight subsets may have an arbitrary positive nonzero size $\left\|W_{c}\right\|_{0}$.

In order to obtain the activation values $A_{c}$, a forward pass of the network is required, since activations are dynamic information. Weights are static information, and are immediately available without performing any computation. This distinction allows us to neatly categorize many pruning metrics which have been proposed in the literature.

TABLE I

NOTABLE SALIENCY METRICS USED FOR CHANNEL PRUNING.

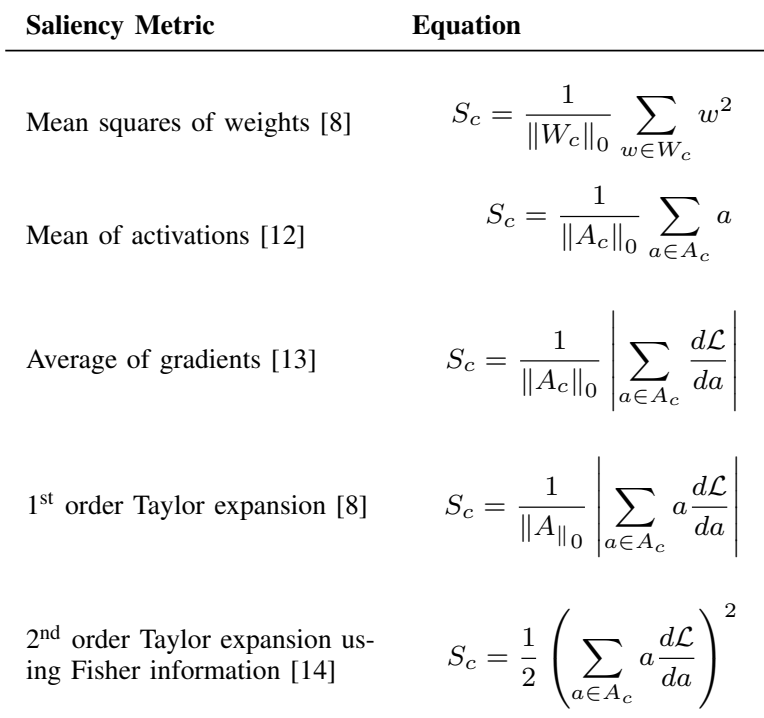

1) Weight-based Saliency Metrics: Commonly used static saliency metrics are the L1-norm of the weights [3] and the mean squares of the weights[8] (see Equation 1). The L2 and L1 norm of the weights have been used in multiple pruning schemes [15], [16], [17] for different granularities of pruning. Weights-based saliency metrics assume that weights of lower magnitude have a lower contribution to the network.

2) Activation-based Saliency Metrics: More recent work has proposed dynamic saliency metrics, which can exploit the information in the activations and gradients. These can only be obtained by performing a forward, and an additional backward, pass of the network, respectively.

Some examples of effective dynamic saliency metrics are the absolute-percentage-of-zeros [4], mean [12] (Equation 2) and standard deviation of activations [18]. Activation-based saliency metrics exploit information only obtainable during forward passes of the network.

3) Gradient-based Saliency Metrics: Conversely we can find saliency metrics that make use of only the gradients [9] such as the use of the average of the gradients, [13] (Equation 3).

However, the information contained in the gradients is often coupled with the activations. The $2^{\text {nd }}$ order Taylor expansion using Fisher information [14] (Equation 5) and $1^{\text {st }}$ order Taylor expansion [8] (Equation 4) are two notable examples of saliency metrics combining both the information of the activations and their gradients.

\section{B. Metric Assumptions}

Most of these saliency metrics rely on some assumptions. For example, when using the L1-norm of weights, the assumption is that smaller weights contribute less to the network. Their underlying assumptions can sometimes be conflicting. The $2^{\text {nd }}$ order Taylor expansion using Fisher information and $1^{\text {st }}$ order Taylor expansion are both derived using the Taylor expansion presented in Figure 1. However, they are constructed under different assumptions. The construction of the $2^{\text {nd }}$ order Taylor Expansion Using Fisher Information [14] assumes that the gradients of the weights and activations are insignificant. Hence, the $1^{\text {st }}$ order terms in Figure 1 are ignored and the second order terms are approximated to the Fisher information to derive Equation 5. On the other hand, when using a first order Taylor expansion, the higher order terms are considered insignificant, meaning we ignore the $2^{\text {nd }}$ order terms in Figure 1 to derive Equation 4.

$$
\mathcal{L}\left(A-A_{c}\right)-\mathcal{L}(A) \approx \underset{1}{2} \frac{d \mathcal{L}}{d A}\left(-A_{c}\right)+\frac{1}{2}\left(-A_{c}\right)^{T} \frac{d^{2} \mathcal{L}}{d A^{2}}\left(-A_{c}\right) !
$$

Fig. 1. Estimating the effect of pruning the $c^{\text {th }}$ output channel of a network with loss function $\mathcal{L}$ using a $2^{\text {nd }}$ order Taylor development around $A$, the activations of the network.

This is a crucial distinction, because these built-in assumptions in the construction of the metrics are typically not simultaneously true for any given network. Moreover, as the pruning process continues, the degree of significance of different components can, and does, change. When most of the remaining information is in higher order components, metrics using only first order components are effectively making random decisions, and vice versa.

For example, in the case of pruning a partially converged network, the gradients of the weights and activations are very unlikely to be negligible. When pruning a fully converged network, the gradients are much more likely to be negligible. When pruning a fully trained network, we start with converged weights. However, as the pruning process proceeds, we may end up pruning partially converged weights, since the pruning process degrades the network.

\section{Composing SAliency Metrics}

When using any one saliency metric for the entire pruning process, we run the risk of the metric assumptions being invalidated, leading to poor decisions being made by the metric. Ideally we could combine the best aspects of different saliency metrics. The chief difficulty lies in combining the numerical output of different pruning metrics, which are not directly comparable.

Consider the application of two saliency metrics $A$ and $B$ to neural network $\mathcal{N}$ with a total of four weights $[a, b, c, d]$. Let us suppose that ranking the weights with each metric yields the rankings $A(\mathcal{N})=[0.5,0.4,0.9,0.1]$ and $B(\mathcal{N})=$ 


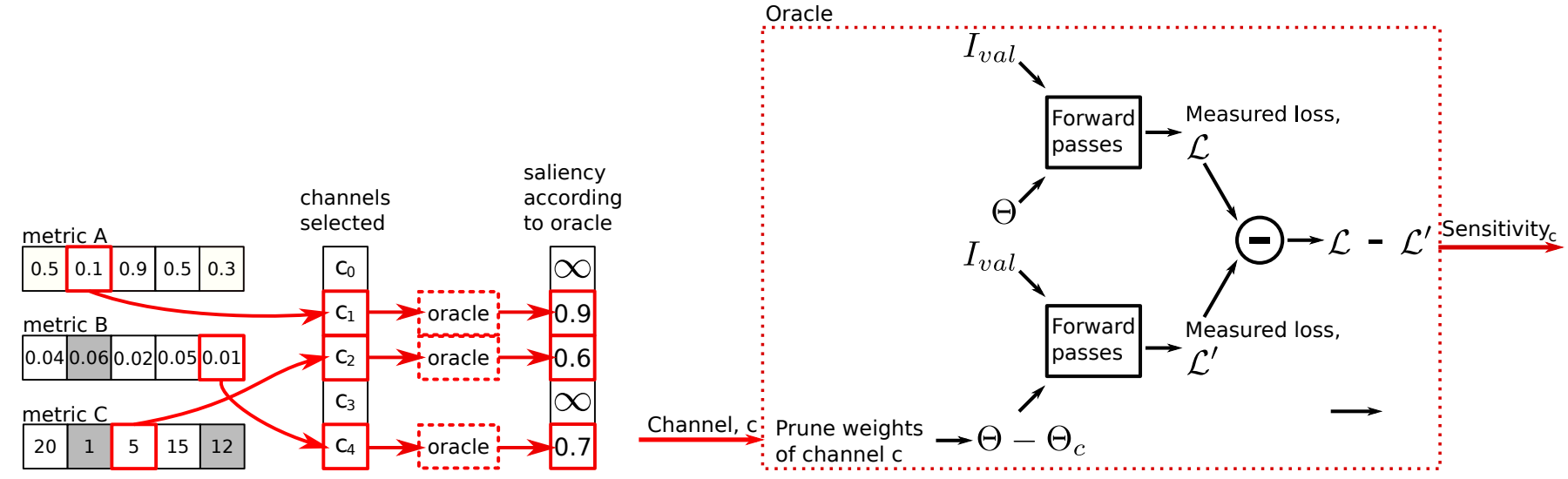

(a) Channel selection $(k=3)$ and oracle evaluation.

(b) Sensitivity evaluation by the oracle for a channel, $c$, according to Equation 6 .

Fig. 2. Combining rankings of saliency metrics $\mathrm{A}, \mathrm{B}$ and $\mathrm{C}$ using a myopic oracle with $k=3$.

$[0.01,0.05,0.04,0.06]$. Metric $A$ indicates removing weight $d$ will have the least effect, while metric $B$ indicates removing weight $a$ will have least effect.

If the metrics agree on the weights to be removed, there is no issue. However, if they disagree, there are two alternatives to consider. If either choice results in the same amount of damage to the network we can call the disagreement trivial. However, if one causes more damage than the other, the disagreement is nontrivial. If we continue to use the suboptimal metric to guide the process, we will introduce more and more relative error.

Inspection of the numerical saliency values assigned to each weight or set of weights by metrics $A$ and $B$ exposes the difficulty of combining these metrics numerically. Although each metric ranks the weights or set of weights in the network, in principle, these saliency values can have arbitrary scales. If we were to combine the metrics with a simple linear combination, such as a weighted average, one metric would be disproportionately selected. As we add more and more metrics to the set, the difficulty increases. However, by performing a forward pass of the network, we may determine at any point the true effect on the loss function of making a particular pruning decision. This is the key to our proposed approach.

\section{A. Our Proposed Method: Myopic Oracle}

When different saliency metrics yield different rankings for the same sets of weights, we can evaluate which ranking is the most correct by performing a direct measurement of the sensitivity of the network to the removal of the proposed subsets of parameters.

For brevity of presentation, in the following treatment we chose subsets of parameters corresponding to whole output feature maps (i.e. channel pruning). However, the approach is no different for other subsets of weights (filters, individual weights or any other granularity of pruning).

For a CNN characterised by the loss function $\mathcal{L}$ and permanent weights $\Theta$, the sensitivity of the $c^{t h}$ channel of the network, using forward passes on the validation set $I_{\text {val }}$, is given by the change in the loss caused by replacing all the weights of the $c^{\text {th }}$ channel $\Theta_{c}$ with zeros as given in Equation 6.

In the case of channel pruning, $\Theta_{c}$ denotes all the parameters that need to be removed to remove a channel and still end up with a dense network. Hence, $\Theta_{c}$ contains $W_{c}$ but may include parameters from other layers that interact with $c^{t h}$ channel.

$$
\text { Sensitivity }_{c}=\mathcal{L}\left(\Theta-\Theta_{c}, I_{\text {val }}\right)-\mathcal{L}\left(\Theta, I_{\text {val }}\right)
$$

At every pruning step, the myopic oracle measures the sensitivity of only $k$ different channels using the validation set. Notionally, $k$ is the number of channels that the myopic oracle can "see". The choice of the value of $k$ depends on the pruning scheme used, but must be at least the number of channels that the pruning scheme considers pruning simultaneously. Hence, $k$ can vary depending on the pruning scheme.

It should be noted that the different consituent saliencies and the sensitivity computed by the myopic oracle use the same dataset, $I_{v a l}$, containing $N_{v a l}$ batches of images. The cost of running the myopic oracle for one channel is similar to the cost of computing the dynamic heuristics that use forward passes only. Hence, if $N_{\text {val }}$ batches are used to measure the sensitivity for each channel the cost of running the oracle (excluding the cost of computing the individual saliencies) is $k \times N_{\text {val }} \times$ cost of forward pass. Assuming that the cost of a forward pass is roughly equal to the cost of a backward pass, the cost of computing a gradients-based saliency metric also using $N_{v a l}$ batches is $2 \times N_{\text {val }} \times$ cost of forward pass. The cost of the myopic oracle is hence not prohibitive but needs to be factored when choosing $k$. A wider view may yield better results but at an increased computational cost.

The myopic oracle visits each of the constituent saliency metrics in a round-robin fashion, and selects the lowest ranked channel to add to the set of channels whose sensitivity should be measured. If the lowest ranked channel has already been selected by another constituent, the second lowest is used 
instead, and so on. This process continues until $k$ unique channels have been selected. The sensitivity of each channel is then tested, yielding the true ranking of these $k$ channels.

Note that the actual saliency values output by each saliency metric are never consumed by the oracle: only the implied ordering of the channels is used. In this way, the oracle is agnostic to the scales of the individual pruning metrics.

Figure 2(a) illustrates selection of channels to be evaluated by the oracle in the case of a pruning scheme with $k=3$. The selected channels then have their sensitivities measured by the oracle according to Equation 6 and Figure 2(b).

\section{B. Constituent Saliency Metrics}

Our composite approach can be used with any saliency metric which can be expressed as a function of weights and activations (including all gradients, which are derivatives of one with respect to the other). However, composing all published saliency metrics following this schema would be unrealistic. Instead we choose a sample of prominent saliency metrics from the literature that perform well in practice. These metrics rely on different kinds of information. We consider both static and dynamic saliency metrics.

We selected the constituent saliency metrics shown in Table I to be combined via the myopic oracle. Prior work has shown each of these saliency metrics are very effective.

Even though they are known to perform well, the chosen saliency metrics are constructed under different assumptions and use a diverse selection of parameters from the network.

\section{EXPERIMENTAL Setup}

For our experimental setup, we chose a set of constituent saliency metrics in Table I to compose via the myopic oracle, and also a general pruning scheme to follow. Simple pruning schemes rely heavily on the saliency metric's prediction whereas in sophisticated schemes[19], [20], [21], [22], the contribution of the saliency metric can become obfuscated by other factors.

\section{A. Choice of Pruning Scheme}

Since our objective is specifically to study the differences in pruning metrics, we chose to eliminate confounding factors by using a simple, iterative pruning scheme without finetuning or retraining. The only change we make to the network weights is to set pruned weights to zero. Using a pruning scheme with retraining is needed to find the absolute best network, however introducing retraining introduces more stochasticity in the results. Since our aim is not to find the best network but the best saliency metric, retraining can obsfucate the results.

Even when retraining is in use, saliency metrics which cause less deviation from the initial test accuracy can lead to less time being spent on retraining, and also to large groups of channels being simultaneously removed, in the case of pruning schemes that allow for simultaneous pruning of multiple channels. Hence, a better saliency metric will always reduce the total amount of effort used to produce pruned networks. Algorithm
1 outlines the simple pruning scheme used to evaluate the myopic oracle.

$\overline{\text { Algorithm } 1 \text { Evaluating different channel selections for a CNN }}$ with loss function $\mathcal{L}$, accuracy $\mathcal{Y}$ and converged weights $\Theta$ with $M$ channels for a user-defined maximum drop in initial test accuracy, maxTestAccDrop

initialTest Acc $\leftarrow \mathcal{Y}\left(\Theta, I_{\text {test }}\right)$

repeat

$S_{c} \leftarrow$ computeSaliency $\left(\mathcal{L}, \Theta, \Theta_{c}, I_{\text {val }}\right)$ for $c \in$ $\{0 \ldots M-1\}$

Get $j$, such that $j=\underset{c \in\{0 \ldots M-1\}}{\operatorname{argmin}} S_{c}$ and $\Theta_{j}$ is a non-zero vector.

$\Theta \leftarrow \Theta-\Theta_{j}$

test $A c c \leftarrow \mathcal{Y}\left(\Theta, I_{\text {test }}\right)$

until testAcc < initialTestAcc - maxTestAccDrop

We iteratively recompute the channel which should be removed, and remove one channel at a time from the entire network until the test accuracy is degraded beyond a certain threshold.

1) CNN Models: LeNet-5 [23] and AlexNet [24] are modified so that the first convolutions accept $32 \times 32$ RGB input images and classify the images between 10 categories. ResNet-20 [25], NIN [26] and the CIFAR10 [27] network are used according to their original descriptions for the CIFAR10 dataset. We also adapted ResNet-20, NIN and AlexNet for the CIFAR-100 [27] dataset. The networks used are trained from scratch using Caffe [28].

TABLE II

SUMMARY OF TRAINED NETWORK ACCURACY ON CIFAR-10 AND CIFAR-100.

\begin{tabular}{l|lllll} 
Network & LeNet-5 & CIFAR10 & ResNet-20 & NIN & AlexNet \\
\hline $\begin{array}{l}\text { Accuracy } \\
\text { (CIFAR-10) }\end{array}$ & $69 \%$ & $73 \%$ & $88 \%$ & $88 \%$ & $84 \%$ \\
$\begin{array}{l}\text { Accuracy } \\
\text { (CIFAR-100) }\end{array}$ & - & - & $59.2 \%$ & $65.7 \%$ & $54.2 \%$ \\
$\begin{array}{l}\text { Convolution } \\
\text { weights }\end{array}$ & $26.5 \mathrm{~K}$ & $79.2 . \mathrm{K}$ & $270 \mathrm{~K}$ & $966 \mathrm{~K}$ & $2.3 \mathrm{M}$
\end{tabular}

2) Experimental Setup and Hyperparameters: We use the CIFAR-10 and CIFAR-100 datasets in our experiments using their respective $50 \mathrm{~K} / 10 \mathrm{~K}$ train/test images. We first train all the networks to convergence using the full training set. The accuracy of our trained networks are shown in Table II. We then prune these fully trained networks. Pruning decisions are made using a subset of the training images, $I_{\text {val }}$. Saliency according to the consituent metrics and myopic oracle are computed using 256 and 512 random images from $I_{\text {val }}$ for CIFAR-10 and CIFAR-100 networks respectively. The top-1 accuracy of the pruned networks are measured using the entire test set, $I_{\text {test }}$. The myopic oracle is evaluated with $k=5,8,12,16$. 


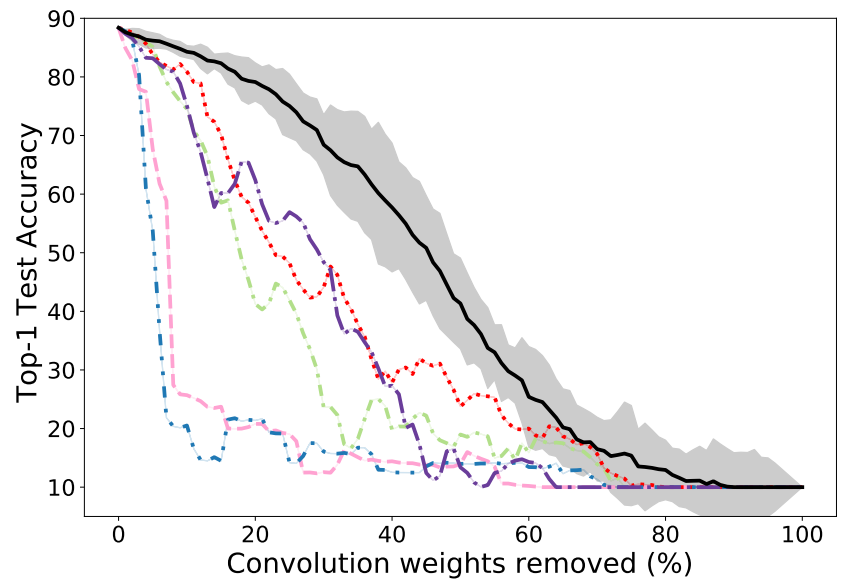

(a) ResNet-20

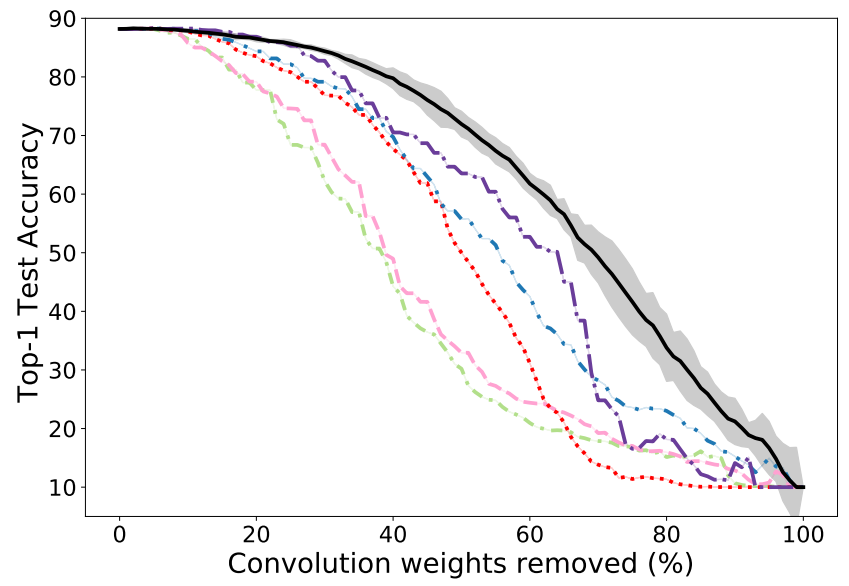

(b) NIN

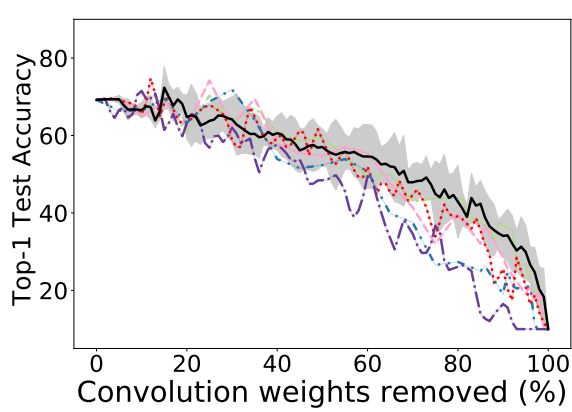

(c) LeNet-5

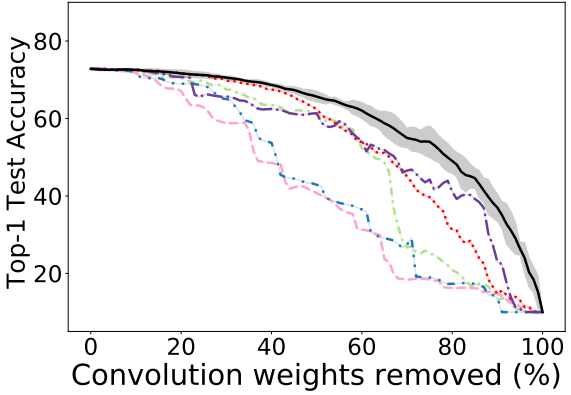

(d) CIFAR10

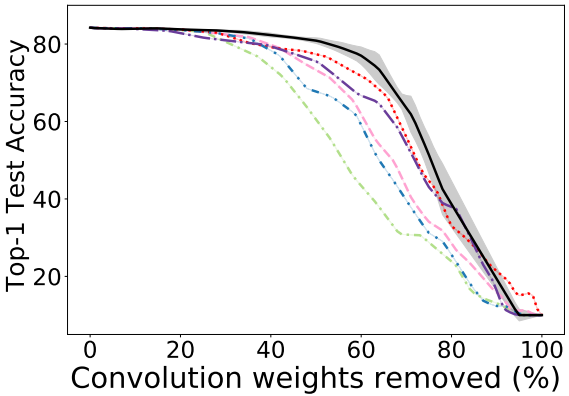

(e) AlexNet

$\begin{array}{lll}- \text { Mean of activations (Equation 2) - " Fisher information (Equation 5) } & \text { Mean squares of weights (Equation 1) } \\ -=1 \text { - } & \text { - Average of gradients (Equation 3) }\end{array}$

Fig. 3. Graphs show top-1 test accuracy versus number of convolution weights (\%) removed by pruning using the CIFAR-10 dataset. Individual saliency metrics are indicated with dashed lines, and the myopic oracle (with $k=8$ ) is indicated with a solid line. Error bands for the myopic oracle are shown based on a $95 \%$ confidence interval for 8 runs of the experiment.

\section{EXPERIMENTAL RESULTS AND DISCUSSION}

Figure 3 presents the result of our experimental evaluation on the five chosen convolutional neural networks. For all five networks, we see that the composite saliency metric matches or exceeds the predictive quality of any of the individual constituent metrics until the test accuracy of the network drops far below useful levels.

\section{A. Behaviour of Composite Metrics}

We would like to draw attention to the ResNet-20 (Figure 3(a)) and NIN (Figure 3(b)) networks in particular. For ResNet-20 (Figure 3(a)), our experiment shows clearly that some saliency metrics are very badly suited for guiding pruning on this network. It is not that these are bad metrics; on the contrary, they perform well on other networks.

However, the assumptions baked into these metrics are at odds with the reality of the relationships of the weights, activations, and gradients in ResNet-20, causing them to severely mispredict the effect on the loss function of pruning any individual channel.Using the myopic oracle allows these metrics to be excluded until their assumptions become more in line with the reality of the network structure, instead of causing pathological behaviour if used indiscriminately.

For NIN (Figure 3(b)), our experiment shows that the composition of metrics via the oracle exhibits smooth, predictable behaviour, where the individual metrics differ dramatically.

Even though the individual metrics have such large differences, the composition of the metrics with the oracle is well-behaved, leading to a much less damaging pruning that with any of the metrics individually.

The remainder of the networks exhibit similar behaviour. For AlexNet (Figure 3(e)), we see again that the composition of the saliency metrics via the oracle yields a smooth, wellbehaved metric, even though the constituent metrics have large differences.

\section{B. Impact of $k$}

From Table III, we can see that using a myopic oracle can lead to a significant increase in the maximum number of 
TABLE III

CONVOLUTION WEIGHTS (\%) REMOVED FOR A 5\% ACCURACY DROP ON CIFAR-10 (GRAY) AND CIFAR-100.

\begin{tabular}{|c|c|c|c|c|c|}
\hline Saliency Metric & LeNet-5 & CIFAR10 & ResNet-20 & NIN & AlexNet \\
\hline \multirow{2}{*}{ Mean of activations } & $24 \pm 0.1$ & $29 \pm 2$ & $6 \pm 1$ & $14 \pm 1$ & $37 \pm 6$ \\
\hline & - & - & $2.6 \pm 0.5$ & $38.1 \pm 0.2$ & $22 \pm 0.2$ \\
\hline \multirow{2}{*}{$1^{\text {st }}$ order Taylor expansion } & $33 \pm 6$ & $16 \pm 0.1$ & $2 \pm 0.1$ & $14 \pm 1$ & $49 \pm 6$ \\
\hline & - & - & $2.3 \pm 0.3$ & $38.2 \pm 0.1$ & $47 \pm 0.4$ \\
\hline \multirow{2}{*}{$\begin{array}{l}2^{\text {nd }} \text { order Taylor expansion } \\
\text { using Fisher information }\end{array}$} & $22 \pm 5$ & $39 \pm 2$ & $6 \pm 3$ & $21 \pm 2$ & $43 \pm 6$ \\
\hline & - & - & $1.1 \pm 0.1$ & $38.5 \pm 0.4$ & $52 \pm 6$ \\
\hline \multirow{2}{*}{ Average of gradients } & $25 \pm 6$ & $24 \pm 4$ & $3 \pm 0.3$ & $24 \pm 1$ & $46 \pm 9$ \\
\hline & - & - & $2.1 \pm 0.3$ & $36.7 \pm 0.3$ & $50 \pm 7$ \\
\hline \multirow{2}{*}{ Mean squares of weights } & 17 & 23 & 5 & 28 & 49 \\
\hline & - & - & 1.7 & 40.7 & 45 \\
\hline \multirow{2}{*}{$k=5$} & $29 \pm 5$ & $37 \pm 4$ & $9 \pm 4$ & $31 \pm 2$ & $52 \pm 0.3$ \\
\hline & - & - & $3.2 \pm 0.3$ & $39.5 \pm 0.4$ & $55 \pm 7$ \\
\hline \multirow{6}{*}{ Myopic Oracle } & $26 \pm 3$ & $40 \pm 3$ & $10 \pm 4$ & $31 \pm 2$ & $53 \pm 5$ \\
\hline & - & - & $3.4 \pm 0.3$ & $39.8 \pm 0.4$ & $57 \pm 7$ \\
\hline & $27 \pm 4$ & $41 \pm 2$ & $11 \pm 4$ & $32 \pm 2$ & $58 \pm 3$ \\
\hline & - & - & $3.7 \pm 0.4$ & $39.9 \pm 0.4$ & $60 \pm 0.8$ \\
\hline & $26 \pm 7$ & $43 \pm 3$ & $11 \pm 3$ & $33 \pm 2$ & $61 \pm 4$ \\
\hline & - & - & $3.7 \pm 0.3$ & $40.0 \pm 0.6$ & $60 \pm 1$ \\
\hline
\end{tabular}

weights but only to a marginal increase when increasing $\mathrm{k}$. This trend would suggest that the channel rankings given by the individual saliency metrics are often accurate. Considering more channels only offers a marginal improvement as the least salient channels are often also ranked lowly by at least one of the constitutent metrics, we only need to determine which saliency metric is accurate for that pruning iteration. Hence, choosing $\mathrm{k}$ to be equal to the number of constitutent metrics allows us to choose between the saliency metrics without inhibitively increasing cost of computation.

\section{Quality of Pruned Networks}

Table III summarizes the level of pruning achieved in our experiments for a maximum reduction of $5 \%$ points in top-1 test accuracy.

Using the myopic oracle to compose existing saliency metrics yields a composite metric which makes better pruning decisions than any of the individual metrics which were composed. The myopic oracle consistently selects channels to prune that result in a smaller loss in test accuracy. We also present the proportion of weights removed for the constitutent saliency metrics, if used exclusively, as in prior work.

On every network, our approach meets or exceeds the performance of all the state of the art saliency metrics used individually. The best results are seen on ResNet-20, where almost twice as many weights can be removed using our approach versus the next-best individual saliency metric.

\section{DISCUSSION}

\section{A. Pruning Scheme}

Combining saliency metrics with a myopic oracle within a simple pruning scheme yields promising results. However, more sophisticated saliency-based pruning schemes can also take advantage of using multiple saliency metrics to remove the maximum number of weights. Our future work will cover testing the use of multiple saliency metrics using a myopic oracle in other pruning schemes.

\section{B. Other Granularities}

The use of multiple saliency metrics is not limited to channel pruning, it can apply to other granularities of pruning. For example, with unstructured pruning (fine-grain pruning) each saliency metric proposes a different set of weights to be removed. The myopic oracle can then choose which proposed set is most accurate.

\section{CONCLUSION}

Our method of composing multiple saliency metrics yields a composite metric that significantly outperforms the individual constituent metrics. By dynamically switching between different metrics based on the actual measured sensitivity of the network, we avoid the occasional poor pruning decision made by even the most advanced saliency metrics. Using our approach, data scientists are freed from having to choose from a dizzying array of potential saliency metrics to guide the pruning process.

By developing a method to dynamically switch between an arbitrary collection of state-of-the-art saliency metrics based on their actual measured performance, we can derive a composite metric with significantly improved performance, pruning up to twice as many weights for the same drop in accuracy in our experiments. Our approach advances the state of the art in identifying unnecessary or redundant sets of neural network parameters.

\section{ACKNOWLEDGEMENT}

This work was supported by Science Foundation Ireland grant 13/RC/2094 to Lero - The Irish Software Research Centre. This work was also partly supported by Arm Research.

\section{REFERENCES}

[1] S. Han, H. Mao, E. Gong, S. Tang, W. J. Dally, J. Pool, J. Tran, B. Catanzaro, S. Narang, E. Elsen, P. Vajda, M. Paluri, W. J. Dally, Dsd: Dense-sparse-dense training for deep neural networks, in: ICLR, 2017. 
[2] S. Han, J. Pool, J. Tran, W. J. Dally, Learning both weights and connections for efficient neural network, in: NIPS, 2015, pp. 1135-1143.

[3] H. Mao, S. Han, J. Pool, W. Li, X. Liu, Y. Wang, W. J. Dally, Exploring the granularity of sparsity in convolutional neural networks, in: 2017 IEEE Conference on Computer Vision and Pattern Recognition Workshops (CVPRW), 2017, pp. 1927-1934. doi:10.1109/CVPRW.2017.241.

[4] H. Hu, R. Peng, Y. Tai, C. Tang, Network trimming: A data-driven neuron pruning approach towards efficient deep architectures, CoRR abs/1607.03250. arXiv:1607.03250. URL http://arxiv.org/abs/1607.03250

[5] Y. LeCun, J. S. Denker, S. A. Solla, Optimal brain damage, in: Advances in Neural Information Processing Systems 2, [NIPS Conference, Denver, Colorado, USA, November 27-30, 1989], 1989, pp. 598-605. URL http://papers.nips.cc/paper/250-optimal-brain-damage

[6] B. Hassibi, D. G. Stork, Second order derivatives for network pruning: Optimal brain surgeon, in: NIPS, Morgan Kaufmann, 1992, pp. 164-171

[7] M. Mozer, P. Smolensky, Skeletonization: A technique for trimming the fat from a network via relevance assessment, in: NIPS, Morgan Kaufmann, 1988, pp. 107-115.

[8] P. Molchanov, S. Tyree, T. Karras, T. Aila, J. Kautz, Pruning convolutional neural networks for resource efficient inference, in: ICLR, 2017.

[9] E. D. Karnin, A simple procedure for pruning back-propagation trained neural networks, IEEE Trans. Neural Networks 1 (2) (1990) 239-242.

[10] J. Frankle, M. Carbin, The lottery ticket hypothesis: Finding sparse, trainable neural networks, in: ICLR, 2019.

[11] N. Lee, T. Ajanthan, P. H. S. Torr, Snip: single-shot network pruning based on connection sensitivity, in: 7th International Conference on Learning Representations, ICLR 2019, New Orleans, LA, USA, May 6-9, 2019, 2019.

URL https://openreview.net/forum?id=B1VZqjAcYX

[12] S. Anwar, K. Hwang, W. Sung, Structured pruning of deep convolutional neural networks, JETC 13 (3) (2017) 32:1-32:18.

[13] C. Liu, H. Wu, Channel pruning based on mean gradient for accelerating convolutional neural networks, Signal Processing 156 (2019) 84 - 91 doi:https://doi.org/10.1016/j.sigpro.2018.10.019. URL http://www.sciencedirect.com/science/article/pii/ S0165168418303517

[14] L. Theis, I. Korshunova, A. Tejani, F. Huszár, Faster gaze prediction with dense networks and Fisher pruning, CoRR abs/1801.05787.

[15] V. Lebedev, V. S. Lempitsky, Fast convnets using group-wise brain damage, in: CVPR, IEEE Computer Society, 2016, pp. 2554-2564.

[16] H. Li, A. Kadav, I. Durdanovic, H. Samet, H. P. Graf, Pruning filters for efficient convnets, in: ICLR, 2017.

[17] Y. He, G. Kang, X. Dong, Y. Fu, Y. Yang, Soft filter pruning for accelerating deep convolutional neural networks, in: IJCAI, ijcai.org, 2018, pp. 2234-2240.

[18] A. Polyak, L. Wolf, Channel-level acceleration of deep face representations, IEEE Access 3 (2015) 2163-2175.

[19] Y. He, J. Lin, Z. Liu, H. Wang, L. Li, S. Han, AMC: automl for model compression and acceleration on mobile devices, in: ECCV (7), Vol 11211 of Lecture Notes in Computer Science, Springer, 2018, pp. 815832.

[20] X. Ding, G. Ding, Y. Guo, J. Han, C. Yan, Approximated oracle filter pruning for destructive CNN width optimization, in: Proceedings of the 36th International Conference on Machine Learning, ICML 2019, 9-15 June 2019, Long Beach, California, USA, Vol. 97 of Proceedings of Machine Learning Research, PMLR, 2019, pp. 1607-1616. URL http://proceedings.mlr.press/v97/ding19a.html

[21] Z. You, K. Yan, J. Ye, M. Ma, P. Wang, Gate decorator: Global filter pruning method for accelerating deep convolutional neural networks, in: Advances in Neural Information Processing Systems 32: Annual Conference on Neural Information Processing Systems 2019, NeurIPS 2019, 8-14 December 2019, Vancouver, BC, Canada, 2019, pp. 2130-2141.

URL http://papers.nips.cc/paper/8486-gate-decorator-global-filter-pruning -method-for-accelerating-deep-convolutional-neural-networks

[22] Z. Wang, C. Li, X. Wang, D. Wang, Towards efficient convolutional neural networks through low-error filter saliency estimation, in: A. C. Nayak, A. Sharma (Eds.), PRICAI 2019: Trends in Artificial Intelligence - 16th Pacific Rim International Conference on Artificial Intelligence, Cuvu, Yanuca Island, Fiji, August 26-30, 2019, Proceedings, Part II, Vol. 11671 of Lecture Notes in Computer Science, Springer, 2019, pp. 255-267. doi:10.1007/978-3-030-29911-8\_20.

URL https://doi.org/10.1007/978-3-030-29911-8 20
[23] Y. Lecun, L. Bottou, Y. Bengio, P. Haffner, Gradient-based learning applied to document recognition, in: Proceedings of the IEEE, Vol. 86, 1998, pp. 2278-2324. doi:10.1109/5.726791.

[24] A. Krizhevsky, I. Sutskever, G. E. Hinton, Imagenet classification with deep convolutional neural networks, Commun. ACM 60 (6) (2017) 84-90. doi: $10.1145 / 3065386$.

URL http://doi.acm.org/10.1145/3065386

[25] K. He, X. Zhang, S. Ren, J. Sun, Deep residual learning for image recognition, in: 2016 IEEE Conference on Computer Vision and Pattern Recognition, CVPR 2016, Las Vegas, NV, USA, June 27-30, 2016, 2016, pp. 770-778. doi:10.1109/CVPR.2016.90.

URL https://doi.org/10.1109/CVPR.2016.90

[26] M. Lin, Q. Chen, S. Yan, Network in network, CoRR abs/1312.4400. arXiv: 1312.4400 URL http://arxiv.org/abs/1312.4400

[27] A. Krizhevsky, Learning multiple layers of features from tiny images (2009).

URL https://www.cs.toronto.edu/ kriz/cifar.html

[28] Y. Jia, E. Shelhamer, J. Donahue, S. Karayev, J. Long, R. Girshick, S. Guadarrama, T. Darrell, Caffe: Convolutional architecture for fast feature embedding, arXiv preprint arXiv:1408.5093. 